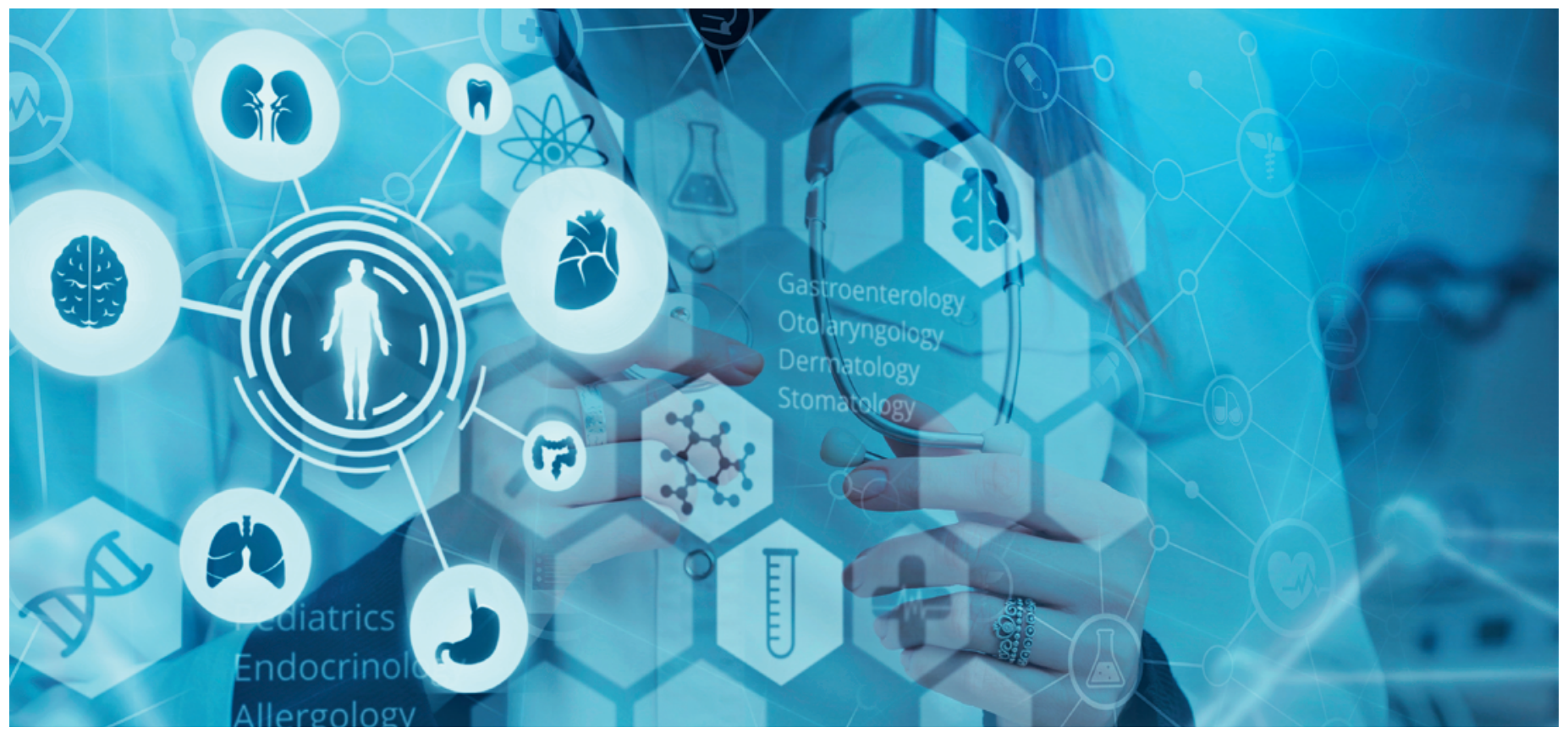

\title{
Das Programm Multimorbidität in der Hausarztmedizin
}

\section{Lilli Herzig ${ }^{a}$, Yolanda Muellera, Andreas Zellerb, Sven Streit ${ }^{c}$, Dagmar M. Haller $^{d}$, Stefan Neuner-Jehle ${ }^{e}$, Nicolas Senn ${ }^{a}$}

a Institut de médecine de famille, Universität Lausanne; ' ${ }^{2}$ Zentrum für Hausarztmedizin, Universität Basel; ${ }^{c}$ Berner Institut für Hausarztmedizin (BIHAM),

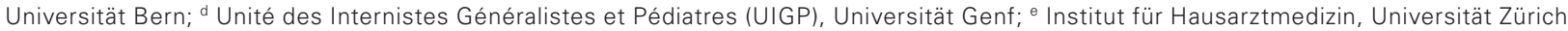

Bedingt durch die Bevölkerungsalterung nimmt die Anzahl der Menschen zu, die an mehreren chronischen Erkrankungen leidet. Multimorbidität wird je nach Studie definiert als die Präsenz von mindestens zwei oder drei chronischen Erkrankungen bei einer betroffenen Person. Multimorbidität ist ein weltweites Kernthema der öffentlichen Gesundheit.

Für die betroffenen Personen kann Multimorbidität mit Schmerzen und eingeschränkter Lebensqualität verbunden sein. Sie erhöht die Sterblichkeit, die Zahl der Krankenhausaufenthalte sowie die Nutzung medizinischer Ressourcen und verursacht hohe Gesundheitskosten. Dennoch hat die medizinische Forschung erst zu Beginn dieses Jahrhunderts angefangen, sich mit der Multimorbidität zu befassen, so dass nur wenige Daten dazu vorliegen, auch wenn die
Multimorbidität gerade in der Hausartzmedizin ein wichtiges Problem darstellt. Tatsächlich steht der HA im Zentrum der Betreuung multimorbider Patienten.

Multimorbidität ist in der Hausarztmedizin eher Regel als Ausnahme.

Es sind die HA, die die Behandlungen in Zusammenarbeit mit anderen Spezialisten koordinieren und den 
Patienten am längsten kennen. Sie sind es auch, die mit den komplexen und einmaligen Konstellationen jedes einzelnen Patienten umgehen müssen, die sich aus gleichzeitig auftretenden häufigen, weniger häufigen und seltenen chronischen Erkrankungen ergeben.

\section{Es sind die Hausärzte, die die Behandlungen in Zusammenarbeit mit anderen Spezialisten koordinieren und den Patienten am längs- ten kennen.}

Es bestehen zahlreiche wissenschaftliche Fragestellungen im Zusammenhang mit der Multimorbidität, die grösstenteils noch nicht beantwortet sind. Dies liegt daran, dass Studien bisher auf einzelne Erkrankungen oder allenfalls auf hoch prävalente Monopatholgien und deren Begleiterkrankungen ausgerichtet waren. Aus diesem Grund wurde 2013 das Forschungsprogramm Multimorbidität in der Hausarztmedizin (MMFM, Multi Morbidité en Médecine de Famille) ins Leben gerufen. Hervorgegangen ist dieses Forschungsprogramm aus einer einzigartigen Zusammenarbeit zwischen fünf Schweizer Universitätsinstituten für Hausarztmedizin (Basel, Bern, Genf, Lausanne und Zürich) unter der Leitung von Frau Doktor Lilli Herzig aus Lausanne. An der Studie beteiligt waren 100 Hausärztinnen und Hausärzte aus der ganzen Schweiz (mit Ausnahme des Tessins) sowie 118 Ärztinnen und Ärzte des epidemiologischen Überwachungssystems Sentinella. Untersucht wurden Definitionen der Multimorbidität, die sich aus Sicht der Wissenschaft, der Politik, der Mitarbeitenden des Gesundheitswesens oder der Patientinnen und Patienten erheblich voneinander unterscheiden können. Dabei interessierte uns, welche Arten von Multimorbidität wie häufig vorkommen, welche Faktoren die Krankheitsbelastung oder die Behandlung beeinflussen und wie die Mittel der Versorgung eingesetzt werden. Dank der Studie verstehen wir nun besser, was die Multimorbidität für die Hausarztmedizin in der Schweiz bedeutet.

Tatsächlich ist Multimorbidität in der Hausarztmedizin eher Regel als Ausnahme und sie nimmt mit dem Alter zu [1]. Aus der Prävalenz Studie "Sentinella» (118 Hausärztinnen und Hausärzte, 2904 Patientinnen und Patienten, Durchschnittsalter 56 Jahre, 44\% Männeranteil) unseres Forschungsprogramms ergeben sich folgende Zahlen: 52\% der hausärztlich behandelten Patienten leiden unter mindestens 2 chronischen Erkrankungen, 35\% unter 3 oder mehr chronischen Erkrankungen, wobei kein signifikanter Geschlechterunterschied besteht. MM existiert selbst bei jungen Menschen und die Prävalenz nimmt mit dem Alter zu: zwischen 21 und 40 Jahren: 19\%; zwischen 41 und
60 Jahren: 45\%; zwischen 61 und 80 Jahren: 72\% und bei mehr als 81 Jahren: $86 \%$ (Zahlen für zwei chronische Erkankungen). Zu den häufigsten chronischen Erkrankungen zählen kardiovaskuläre Erkrankungen (43\%, vor allem Bluthochdruck), psychologische Probleme (29\%, vor allem Depression) und Stoffwechselerkrankungen (24\%) [2].

Unter Mithilfe von 100 Schweizer Hausärztinnen und Hausärzten wurde im Rahmen unseres Forschungsprogramms eine Querschnittstudie durchgeführt, die sich mit dem Blickwinkel von Hausarzt und multimorbidem Patient beschäftigt. An dieser Studie waren 888 multimorbide Patienten beteiligt, die unter mindestens 3 chronischen Erkrankungen litten. Diese Patientinnen und Patienten sind ihrem Hausarzt durchschnittlich seit 11 Jahren bekannt, weisen maximal 19 gleichzeitige chronische Erkrankungen auf und nehmen im Durchschnitt 7 Medikamente täglich ein. Nur 10\% dieser Patienten erhalten häusliche Pflege durch Spitex, was die zentrale Bedeutung des Hausarztes belegt. An der Versorgung von $84 \%$ der Patienten sind weitere Fachärzte beteiligt, durchschnittlich 1,9 Fachärzte pro Patient. Interessant ist die Feststellung, dass die Anzahl der beteiligten Fachärzte bis zum Alter von 80 Jahren zu-, danach aber wieder abnimmt, weshalb hochbetagte Patientinnen und Patienten häufig nur noch durch den Hausarzt behandelt werden. Die Patienten hatten im Durchschnitt im Laufe der letzten 12 Monate 12,9 Arzttermine (SD 8,8), wobei die Zahl der Termine pro Jahr zwischen 1 und 80 variiert.

\section{2\% der hausärztlich behandelten Patienten leiden unter mindestens 2 chronischen Erkran- kungen.}

Die in der Querschnittstudie hochprävalenten Erkrankungen (definiert als $>5 \%$ unseres Studienkollektivs) entsprechen dem, was bereits aus der medizinischen Literatur bekannt ist. Diese Erkrankungen können assoziiert auftreten (patterns). Unsere Studie ermöglichte es, die vier häufigsten Assoziationen zu beschreiben: 1. Kardiovaskuläre Probleme und Risikofaktoren; 2. Altersbedingte chronische Erkrankungen und endokrine Erkrankungen (Schwerhörigkeit, Katarakte, Diabetes usw.); 3. Abhängigkeit von Alkohol oder Tabak und 4. Knochen- und Gelenkschmerzen sowie psychische Probleme [3]. Diese Assoziationen gleichen stark dem, was weltweit in der Literatur beschrieben wird [4, 5]. Unsere Studie zeigt aber auch, dass neben diesen gut beschriebenen und hochprävalenten chronischen Erkrankungen noch eine ganze Reihe weiterer Erkrankungen existiert, die weniger häufig oder auch selten auftreten, die der Hausarzt beziehungsweise die Haus- 
ärztin aber bei der allgemeinärztlichen Versorgung berücksichtigen muss. Und unter dieser Perspektive sind die Kombinationen fast unendlich. Diese selteneren chronischen Erkrankungen machen Multimorbidität zu einem hochkomplexen Problem, was erklärt, weshalb für bestimmte Konstellationen häufig keine klinischen Empfehlungen verfügbar sind.

\section{Hausärztinnen und -ärzte müssen ihr ganzes medizinisches Können aufwenden, um klini- sche Empfehlungen und Leitlinien anzupassen.}

Unsere verschiedenen Analysen haben auch gezeigt, dass eine sehr starke Assoziation zwischen Multimorbidität und einer durch die Patienten angegebenen signifikant geringeren Lebensqualität besteht [6]. Ausserdem haben uns die mit Multimorbidität einhergehenden Faktoren interessiert. So sind die drei Deprivationsaspekte (gesundheitliche Deprivation, soziale Deprivation oder materielle Deprivation) bei multimorbiden Patienten deutlich grösser, wobei es abhängig vom Alter zu Abweichungen kommt: Die soziale Deprivation ist bei der Bevölkerungsgruppe über 64 Jahre höher, während jüngere Patienten vor allem unter materieller und gesundheitlicher Deprivation leiden [7]. Ein weiterer wichtiger Faktor, der in unserer Studie untersucht wurde und mit Multimorbidität zusammenhängt, ist die Gesundheitskompetenz [8].

\section{Schlussfolgerung}

Der Hausarzt spielt eine zentrale Rolle bei der Behandlung multimorbider Patienten: Er koordiniert häufig zahlreiche Beteiligte, wobei die Zahl der Fachärzte mit der Zahl der Erkrankungen zu-, mit dem Alter der Patienten aber wieder abnimmt. Hochprävalente chronische Erkrankungen kommen auch in der Hausarztmedizin häufig vor, aber die Multimorbidität beschränkt sich nicht auf hochprävalente chronische Erkrankungen. Vielmehr ist das Auftreten von chronischen Erkrankungen mittlerer oder geringer Prävalenz und allen damit möglichen Kombinationen eher die Norm denn die Ausnahme. Somit müssen Hausärztinnen und -ärzte ihr ganzes medizinisches Können aufwenden, um klinische Empfehlungen und Leitlinien anzupassen. Es scheint daher speziell wichtig die Gesundheitsobjektive in Absprache mit dem Patienten zu priorisieren. Nur so erhalten wir eine kohärente Behandlung und können das Dogma der Monopathologien (oder deren Ueberlagerung) überwinden. Zahlreiche Faktoren hängen mit der Multimorbidität zusammen, wie etwa Lebensqualität, Deprivation oder Gesundheitskompetenz. Die Definition von Multimorbidität unterscheidet sich je nach Betroffenem (Patient, Angehörige, Pflegepersonal, behandelnder Arzt, andere Fachärzte, Versicherungen oder Politiker) - daher müssen diese verschiedenen Blickwinkel berücksichtigt werden, um sich ein umfassenderes Bild zu verschaffen.

\section{Bei folgenden Personen und Einrich- tungen möchten wir uns bedanken}

Bei den Master-Studentinnen und -Studenten der Universität Lausanne für ihre im Rahmen dieses Programms durchgeführten qualitativen Studien.

Bei den 100 Ärztinnen und Ärzten, die an der MMFMStudie teilgenommen haben, sowie bei ihren Patientinnen und Patienten.

Bei den 118 Ärztinnen und Ärzten des SentinellaSystems, die an der Prävalenz-Studie teilgenommen haben.

Bei den Mitgliedern der SAFMed (Swiss Academy of Family Medicine), die dieses umfassende Projekt unterstützt und zu ihm beigetragen haben, und bei der Schweizerischen Universitätskonferenz für die finanzielle Unterstützung.

Bildnachweis

(c) Ravil Sayfullin | Dreamstime.com

Literatur

1 Barnett K, Mercer SW, Norbury M, Watt G, Wyke S, Guthrie B. Epidemiology of multimorbidity and implications for health care, research, and medical education: a cross-sectional study. Lancet. 2012;380(9836):37-43.

2 Excoffier S, Herzig L, N'Goran AA, Deruaz-Luyet A, Haller DM. Prevalence of multimorbidity in general practice: a cross-sectional study within the Swiss Sentinel Surveillance System (Sentinella). BMJ open. 2018;8(3):e019616.

3 Deruaz-Luyet A, N'Goran AA, Senn N, Bodenmann P, Pasquier J, Widmer D, et al. Multimorbidity and patterns of chronic conditions in a primary care population in Switzerland: a cross-sectional study. BMJ open. 2017;7(6):e013664.

4 Jovic D, Vukovic D, Marinkovic J. Prevalence and Patterns of MultiMorbidity in Serbian Adults: A Cross-Sectional Study. PloS one. 2016;11(2):e0148646.

5 Marengoni A, Winblad B, Karp A, Fratiglioni L. Prevalence of chronic diseases and multimorbidity among the elderly population in Sweden. American journal of public health. 2008;98(7):1198-200.

6 N'Goran AA, Deruaz-Luyet A, Haller DM, Zeller A, Rosemann T, Streit S, et al. Comparing the self-perceived quality of life of multimorbid patients and the general population using the EQ-5D-3L. PloS one. 2017;12(12):e0188499.

7 Leiser S, Deruaz-Luyet A, N'Goran AA, Pasquier I, Streit S, NeunerJehle S, et al. Determinants associated with deprivation in multimorbid patients in primary care - A cross-sectional study in Switzerland. PloS one. 2017;12(7):e0181534.

8 N'Goran AA, Pasquier J, Deruaz-Luyet A, Burnand B, Haller DM Neuner-Jehle S, et al. Factors associated with health literacy in multimorbid patients in primary care: a cross-sectional study in Switzerland. BMJ open. 2018;8(2):e018281 